\title{
Tecnologías de remoción de plaguicidas presentes en
}

\section{aguas}

\section{Removal technologies of pesticides present in water}

\author{
Rodrigo Andrés Sarria-Villa $*^{1(\mathbb{D})}$, José António Gallo-Corredor ${ }^{1(i)}$ y \\ Edier Humberto Pérez ${ }^{2}$ (D) \\ ${ }^{1}$ Grupo de Investigación en Química Analítica (GIQA), \\ Departamento de Química, Universidad del Cauca, Colombia \\ ${ }^{2}$ Grupo de Agroquímica, Departamento de Química, Universidad del \\ Cauca, Colombia
}

Resumen. Los plaguicidas son conocidos por ser un tipo de sustancias químicas perjudiciales a la humanidad, su desarrollo y aplicación para la protección de las plantas es ahora una medida integrante del sistema de producción vegetal a nivel global. Nueve de las doce sustancias químicas orgánicas más peligrosas y persistentes conocidas sobre el planeta son plaguicidas y sus derivados. Debido a su constante presencia en el medio ambiente, su determinación y cuantificación es realizada principalmente empleando métodos cromatográficos con tratamientos previos de matriz. Gran parte de los plaguicidas tienen como destino final las fuentes hídricas, contaminando y afectando este valioso recurso. Debido a la fuerte naturaleza recalcitrante de los plaguicidas, se han planteado diferentes procesos de descontaminación, dentro de los que se incluyen la precipitación, procesos electroquímicos, intercambiadores iónicos, tratamientos biológicos, procesos avanzados de oxidación, tratamientos con membrana y adsorción, entre otros.

Palabras Claves. Plaguicidas; Contaminación; Tecnologías de remoción; Depuración

\begin{abstract}
Pesticides are known to be a type of chemical substances harmful to mankind, their development and application for the protection of plants is now an integral measure of the plant production system globally. Nine of the twelve most dangerous and persistent organic chemicals known on the planet are pesticides and their derivatives. Due to its constant presence in the environment, its determination and quantification is mainly carried out using chromatographic methods with previous matrix treatments. A large part of the pesticides have as their final destination the water sources, contaminating and affecting this valuable resource. Due to the strong recalcitrant nature of pesticides, different decontamination processes have been proposed, including precipitation, electrochemical processes, ion exchangers, biological treatments, advanced oxidation processes, membrane treatments and adsorption, among others.
\end{abstract}

Keywords. Pesticides; pollution; removal technologies; purification.

Como Citar. R. Sarria-Villa, J. Gallo-Corredor y E. H. Pérez, "Tecnologías de remoción de plaguicidas presentes en aguas", Jou. Cie. Ing., vol. 12, no. 1, pp. 215-229, 2020. doi:10.46571/JCl.2020.1.19

Recibido: 03/04/2019 Revisado: 12/06/2020Ａceptado: 28/07/2020 


\section{Introducción}

Los plaguicidas alcanzan los ecosistemas acuáticos mediante la aplicación directa, dispersión, asperción aérea, erosión y escorrentía superficial o subsuperficial de las tierras agrícolas, o mediante la descarga de los efluentes de las fábricas y aguas residuales [1-3]. El uso de estas sustancias plantea un serio problema de salud, sus propiedades tóxicas incluyen potencia carcinogénica, inmunotoxicidad y una gama de efectos endocrinos relacionados con la reproducción [4]. Los plaguicidas organofosforados (OPs) son ampliamente utilizados en la agricultura para la protección de cultivos y uso forestal, entre otros. Estas moléculas de OPs son tóxicas y actúan sobre la actividad de la acetil-colinesterasa y fueron desarrollados para sustituir a los plaguicidas organoclorados (OCs) que están prohibidos debido a su persistencia en el medio ambiente y a su capacidad de bioacumulación en la cadena alimenticia [5]. Aunque los OCs fueron suspendidos oficialmente desde hace más de 30 años, sus residuos siguen siendo detectados en alimentos, suelos, sedimentos y biota [6-10]. Algunos de los plaguicidas organofosforados como el metil paration y el etil paration son ligeramente persistentes en el medio ambiente [11]. Ellos son compuestos moderadamente apolares a polares y se puede mover desde el suelo por arrastre y lixiviación, lo que conduce a un problema en los depósitos de suministro de agua potable [12]. Los herbicidas tipo triazina forman un amplio grupo de sustancias utilizadas para el control de pre y post-aparición de las plantas que de alguna u otra manera compiten con el cultivo de interés. Este tipo de herbicidas, y sus productos de degradación, son muy tóxicos y altamente resistentes y permanecen muchos años en el agua del suelo, plantas y animales. Existen diferentes métodos para el aislamiento de los plaguicidas del agua y sedimentos tales como Soxhlet, fluidos supercríticos (SFE), la extracción líquida a presión (PLE), la extracción de solvente acelerada (ASE), la extracción asistido por microondas (MAE), extracción en fase sólida (SPE) y la extracción por ultrasonido (USE), micro-extracción en fase sólida (SPME) y dispositivos de membrana semipermeable (SPMDs) entre otras [13,14]. Las técnicas de cromatografía de gases, acoplada a detectores de captura de electrones (ECD) y espectrometría de masas (MS) se utilizan para determinar OCs; los detectores de nitrógeno-fósforo (NPD), fotométrico de llama (FPD), y espectrometría de masas (MS) han sido considerados como los más apropiados para la determinación de OPs en muestras acuosas [15]. La cromatografía de gases (GC) y la cromatografía líquida de alta resolución (HPLC) son buenas opciones para el monitoreo de triazinas en agua y sedimentos [16]. Debido a los potenciales efectos tóxicos de los plaguicidas es necesario analizar e identificar el potencial de riesgo adverso hacia el ambiente y al ser humano para prevenir afectaciones sobre las poblaciones y proteger a las comunidades asentadas en las riberas de los ríos que utilizan sus aguas para diferentes actividades de subsistencia [17]. Se han desarrollado diferentes métodos eficientes para la remoción de plaguicidas, entre los que se encuentran los métodos físicos, químicos y biológicos, los cuales incluyen la adsorción, oxidación, degradación catalítica, filtración por membrana, degradación bacteriana y fúngica y empleo de nanomateriales para la remoción de plaguicidas presentes en aguas residuales [18-20]. En este documento se describen los procesos más importantes para el tratamiento de plaguicidas presentes en matrices acuosas.

\section{Tecnologías de remoción de plaguicidas presentes en aguas}

\subsection{Precipitación}

En la precipitación se busca la eliminación de una sustancia disuelta indeseable, por adición de un reactivo que forme un compuesto insoluble con el mismo, facilitando así su eliminación ya sea por filtración, sedimentación, coagulación-floculación u otro método similar. Se han realizado estudios de remoción de plaguicidas empleando la coagulación [21], obteniéndose porcentajes de remoción promedio del $35 \%$ utilizando diferentes dosis de coagulante (Sulfato de aluminio). Porcentajes de remoción por debajo del 50 \% fueron obtenidos en la mayoría de los casos, excepto 
para plaguicidas como trifluralin, hexaclorobenceno, heptacloro, aldrin e isodrin, para los cuales las eficiencias de remoción estuvieron cerca del $90 \%$ y DDTs, los cuales fueron degradados cerca del $70 \%$.

\subsection{Procesos electroquímicos}

En los procesos electroquímicos se utiliza energía eléctrica como vector de descontaminación provocando reacciones de óxido-reducción tanto en el ánodo como en el cátodo [22]. Su costo es una de las principales desventajas del proceso, sin embargo, como ventajas cabe destacar la versatilidad de los equipos, la ausencia de la utilización de reactivos y la selectividad, pues controlar el potencial del electrodo permite seleccionar la reacción electroquímica dominante deseada. Este método se ha empleado de forma eficiente para eliminar plaguicidas tipo de organofosforados como el paration y metil paration, con reducciones del COD (Chemical Oxygen Demand) hasta del $80 \%$, siendo su principal ventaja el tratamiento de altas concentraciones de plaguicidas presentes en aguas residuales [23]. Los procesos electroquímicos avanzados (EAOPs) han sido resaltados como una potencial alternativa para el tratamiento de aguas, debido a que permiten mineralizar completamente sustancias altamente persistentes como los farmacéuticos, plaguicidas, colorantes azo, ácidos carboxílicos, entre otros. Además de la eficiente remediación de contaminantes orgánicos persistentes (POPs), los EAOPs, también presentan importantes características de significado ambiental como: Suaves condiciones de operación a temperatura y presión ambiental, reactores compactos, no se requieren sustancias químicas auxiliares, no se producen residuos secundarios, fácil de combinar con otras tecnologías de tratamiento, pueden ser fácilmente automatizadas, presentan asequibles costos de operación. Todas estas características, hacen de los EAOPs, tecnologías ambientalmente amigables, con una pequeña huella de carbono. Entre los diferentes tipos de los EAOPs, la oxidación anódica $(\mathrm{AO})$ y la oxidación electroquímica (EO), son los procesos más estudiados debido a su versatilidad y fácil escalamiento [24]. La idea fundamental del proceso electroquímico toma ventaja de las reacciones que ocurren en el ánodo (oxidación de contaminantes) y en el cátodo (reducción de metales pesados) para remover contaminantes, los cuales han sido ampliamente usados para remover metales pesados presentes en solución. Mientras que la aplicación de procesos electroquímicos en la remoción de contaminantes orgánicos reside en la posibilidad de alcanzar una degradación parcial o una completa mineralización por la reacción de oxidación anódica. La oxidación de contaminantes orgánicos en una celda electrolítica puede ocurrir en dos rutas diferentes, ya sea en una oxidación anódica directa o una oxidación indirecta. La oxidación anódica directa o electrólisis ocurren directamente sobre el ánodo $(\mathrm{M})$ e involucra reacciones de transferencia de carga directa entre la superficie del ánodo y el contaminante orgánico involucrado. El mecanismo solamente involucra la mediación de los electrones, los cuales se presentan en la oxidación de algunos contaminantes orgánicos definido por los potenciales de reducción más negativos que el potencial de evolución de reducción del oxígeno (OER). La electrólisis directa normalmente requiere una previa adsorción de los contaminantes sobre la superficie del ánodo, el cual es un proceso que limita la velocidad y no conduce a la combustión de los contaminantes orgánicos. De otro lado, el proceso EO indirecta, es mediado por la electrogeneración in-situ de especies altamente oxidantes generadas desde la superficie del electrodo. Diferentes tipos de especies oxidantes se pueden generar por el proceso de EO, siendo unas de las más importantes, las especies reactivas de oxígeno y las especies activas de cloro [25]. Las reacciones de oxidación electroquímica (EO) por especies reactivas de oxígeno se basa en la electrogeneración del radical hidroxilo $(\bullet \mathrm{OH})$ adsorbido sobre la superficie del ánodo como un intermediario del potencial de reacción de evolución de oxígeno (OER). Como se presenta en la ecuación $1, \mathrm{M}$ representa al ánodo y $M(\bullet \mathrm{OH})$ el radical hidroxilo adsorbido.

$$
M+\mathrm{H}_{2} \mathrm{O} \rightarrow M(\bullet \mathrm{OH})+\mathrm{H}^{+}+e^{-}
$$


Sin embargo, son inevitables las reacciones competitivas que consumen especies radicalarias, conduciendo a que la evolución del oxígeno también sea factible. Ecuaciones 2 y 3 .

$$
\begin{gathered}
M(\bullet \mathrm{OH})+\mathrm{H}_{2} \mathrm{O} \rightarrow \mathrm{M}+\mathrm{O}_{2}+3 \mathrm{H}^{+} 3 e^{-} \\
2 \mathrm{M}(\bullet \mathrm{OH}) \rightarrow 2 \mathrm{M}+\mathrm{O}_{2}+2 \mathrm{H}^{+}+2 e^{-}
\end{gathered}
$$

Para producir grandes cantidades de $M(\bullet O H)$, se deben usar ánodos con un alto sobre potencial para OER que promuevan la reacción (1) y se eviten reacciones parásitas (2 y 3). También se han estudiado diferentes materiales en los ánodos para mejorar la eficiencia de mineralización de los contaminantes. Varios autores proponen clasificar los electrodos de sobrepotencial de evolución de oxígeno para el tratamiento de aguas residuales en dos grupos: ánodos "activos" y "no activos" . La diferencia en la eficiencia de estos ánodos está relacionada con la entalpía de adsorción de radicales $\mathrm{OH}$ sobre la superficie del ánodo. Especies fisioadsorbidas son más oxidantes que las fuertemente quimioadsorbidas, representadas por las ecuaciones 1 y 4 respectivamente. En la figura 1 se presentan el esquema de generación de especies reactivas de oxígeno sobre a) ánodos activos y b) ánodos no activos.

$$
M(\bullet O H) \rightarrow M O+H^{+}+e^{-}
$$

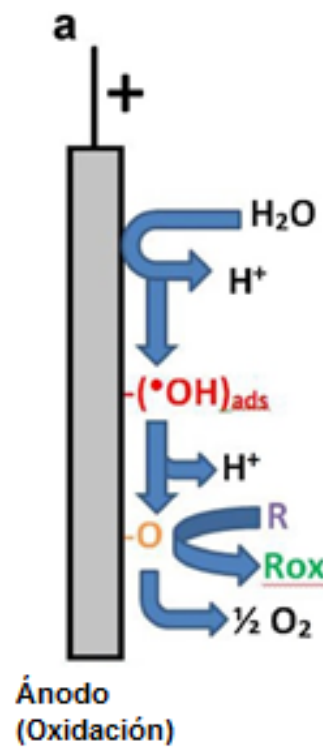

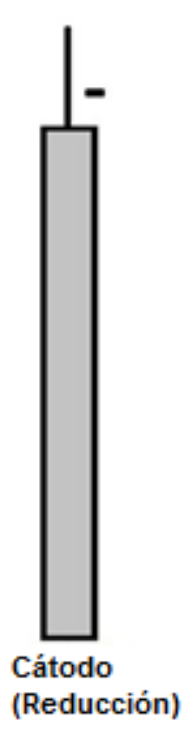

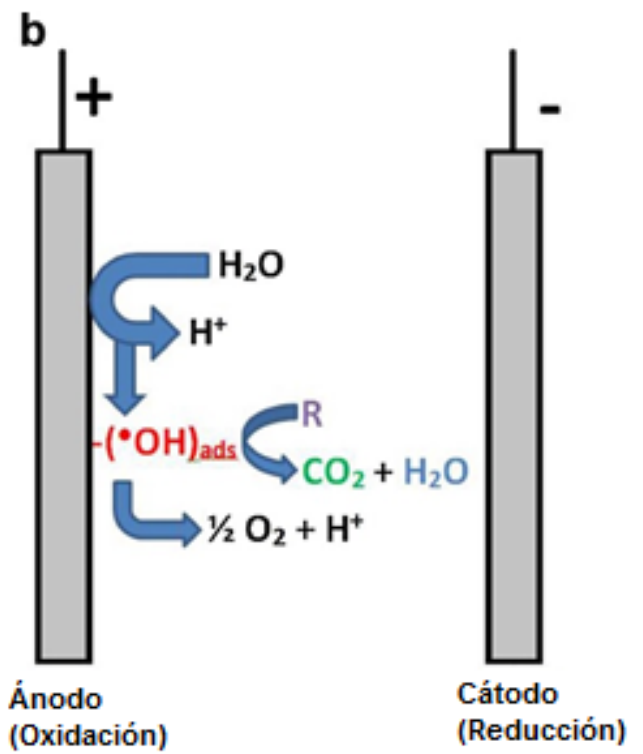

Figura 1: Esquema de generación de especies reactivas de oxígeno sobre a) ánodos activos b) ánodos no activos [25].

La oxidación electroquímica generando in situ especies cloradas activas es otro de los procesos de EO más empleados para remover contaminantes orgánicos. Los principios están relacionados con la oxidación del anión cloruro sobre el ánodo para producir cloro [25].

\subsection{Intercambio iónico}

El intercambio iónico es una operación en la que se utiliza un material, habitualmente denominado resinas de intercambio iónico, que es capaz de retener selectivamente sobre su superficie los iones disueltos en el agua, los mantiene temporalmente unidos a la superficie, y los 
cede frente a una disolución con un fuerte regenerante. Se han empleado resinas de intercambio aniónico combinadas con carbón activado para la eliminación de plaguicidas como la atrazina e isoproturon, mostrando un aumento en la eliminación hasta de un $35 \%$ [26]. En la figura 2 se presenta la preparación de un polímero iónico sobre un nanotubo de carbono para retener el plaguicida imidacloprid y realizar su detección electroquímica.

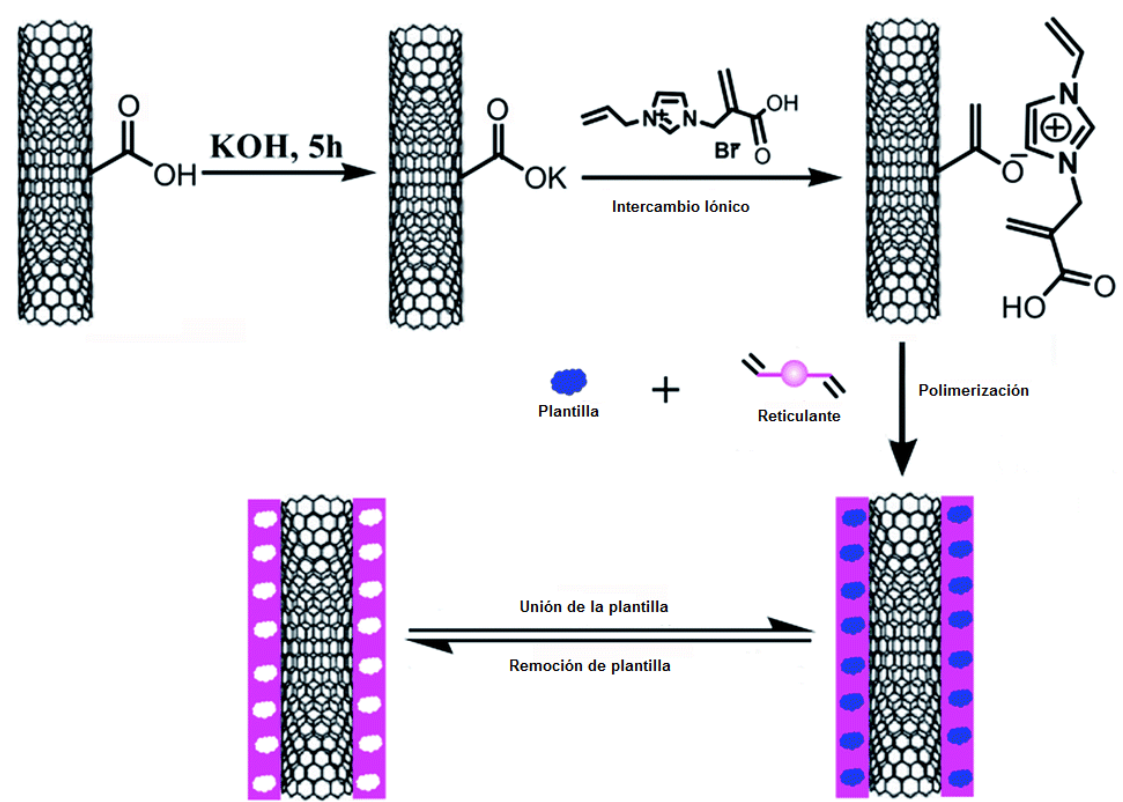

Figura 2: Preparación de una superficie de un polímero iónico sobre un nanotubo de carbono empleado para retener plaguicidas [27].

\subsection{Tratamientos biológicos}

Los tratamientos biológicos constituyen una serie de importantes procesos de tratamiento que tienen en común la utilización de microorganismos (entre las que destacan las bacterias) para llevar a cabo la eliminación de componentes indeseables del agua, aprovechando la actividad metabólica de los mismos sobre esos componentes. De acuerdo al elemento aceptor de electrones en el metabolismo bacteriano el sistema puede ser anaerobio, aerobio o anóxico. Una variedad de procesos fisiológicos son de gran importancia en la biodegradación de plaguicidas. Por ejemplo, algunos plaguicidas degradados pueden mineralizar el plaguicida objetivo a concentraciones ambientales importantes por medio de una vía catabólica, en cambio, otros plaguicidas pueden conducir a compuestos recalcitrantes o intermediarios de biodegradación. También la bioestimulación es una técnica de biorremediación que involucra el aumento de la actividad de la comunidad microbiana. Esto se hace potenciando los sustratos limitados y los nutrientes en el medio ambiente. Por ejemplo, la biodegradación anaeróbica de plaguicidas como la bentazona, mecoprop y diclorprop fue estimulada con la adición de oxígeno para una eliminación de los plaguicidas. Otra forma de aumentar el rendimiento de biodegradación es por medio del enriquecimiento taxonómico de las comunidades microbianas. Este tipo de estrategias pueden ser empleadas con el fin de remover mezclas complejas de plaguicidas presentes en matrices ambientales [28].

\subsection{Procesos avanzados de oxidación}

Los procedimientos avanzados de oxidación se definen como "aquellos procesos de oxidación que implican la generación de radicales hidroxilo en cantidad suficiente para interaccionar 
con los compuestos orgánicos del medio". Se trata de una familia de métodos que utilizan la elevada capacidad oxidante de los radicales $\mathrm{OH}$ y que se diferencian entre sí en la forma en la que los generan $[29,30]$. Los más comunes utilizan combinaciones de ozono $\left(\mathrm{O}_{3}\right)$, peróxido de hidrógeno $\left(\mathrm{H}_{2} \mathrm{O}_{2}\right)$, radiación ultravioleta y fotocatálisis. Los radicales generados durante el proceso producen la mineralización de compuestos orgánicos refractarios, patógenos presentes en el agua y sub-productos de desinfección [31,32]. Por otro lado, se trata de procesos que utilizan reactivos que representan una importante inversión económica tales como el agua oxigenada o el ozono, por lo que su utilización debe restringirse a situaciones en las que otros procesos más baratos, como los biológicos, no sean posibles. Su máximo potencial se aprovecha cuando se consiguen integrar con otros tratamientos, como la adsorción o los tratamientos biológicos, a fin de conseguir la máxima economía de oxidante [33].

Entre los procesos avanzados de oxidación (AOPs), la fotocatálisis heterogénea empleando semiconductores como catalizadores $\left(\mathrm{TiO}_{2}, \mathrm{ZnO}, \mathrm{Fe}_{2} \mathrm{O}_{3}, \mathrm{CdS}, \mathrm{GaP}\right.$ y $\left.\mathrm{ZnS}\right)$ ha demostrado su eficiencia en degradar un amplio rango de sustancias orgánicas refractarias en compuestos biodegradables y eventualmente mineralizados a dióxido de carbono y agua. Entre los catalizadores semiconductores, el dióxido de titanio $\left(\mathrm{TiO}_{2}\right)$ ha recibido gran interés en investigación y desarrollo en tecnología de fotocatálisis bajo la energía del fotón $(\lambda)$ entre 300 nm y $390 \mathrm{~nm}$ y permanece estable después de repetidos ciclos fotocatalíticos, mientras que CdS o GaP son degradados produciendo sustancias tóxicas [34,35]. Otra característica del catalizador de $\mathrm{TiO}_{2}$ es su estabilidad térmica y química o resistencia al rompimiento químico y sus fuertes propiedades mecánicas prometen una amplia aplicación en el tratamiento fotocatalítico de las aguas.

2.5.1. Fotocatálisis heterogénea con $\mathrm{TiO}_{2}$ El semiconductor de $\mathrm{TiO}_{2}$ ha sido ampliamente utilizado como fotocatalizador para inducir una serie de reacciones oxidativas y reductivas sobre su superficie. Cuando la energía del fotón (hv) es mayor o igual a la energía del bandgap del $\mathrm{TiO}_{2}$, usualmente $3.2 \mathrm{eV}$ (anatasa) o $3.0 \mathrm{eV}$ (rutilo), el electrón solo es fotoexcitado a la banda de conducción vacía en femtosegundos. La figura 3 representa el mecanismo de formación del par electrón-hueco.

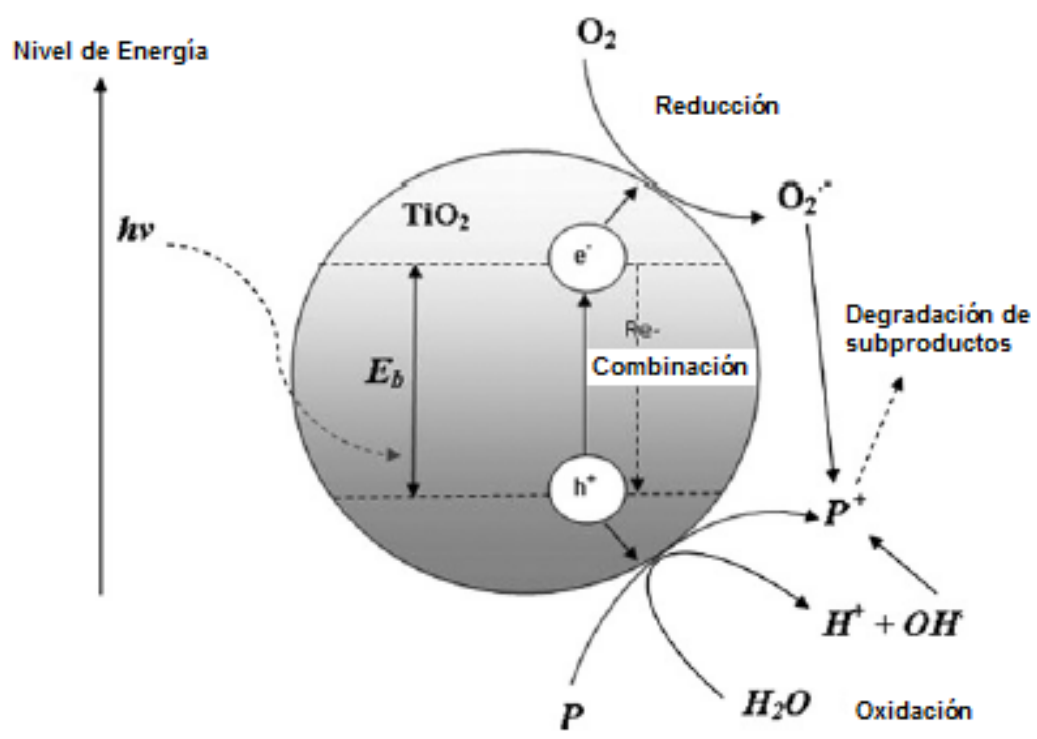

Figura 3: Mecanismo de formación foto-inducida del par electrón-hueco en una partícula de un semiconductor de $\mathrm{TiO}_{2}$ en presencia de un contaminante de agua (P) [36]. 
Cuando la partícula de $\mathrm{TiO}_{2}$ es irradiada con adecuada $\mathrm{h} \nu$. La longitud de onda para cada fotón usualmente corresponde a $\lambda<400 \mathrm{~nm}$. La excitación fotónica conduce a una banda de valencia vacía, creando un par electrón-hueco $\left(\mathrm{e}^{-}-\mathrm{h}^{+}\right)$. La serie de reacciones oxidativasreductivas en cadena pueden ser representadas como sigue:

Fotoexcitación : $\mathrm{TIO}_{2}+h v \rightarrow e^{-}+h^{+}$

Captura portadores de carga $e-: e_{C B}^{-} \rightarrow e_{T R}^{-}$

Captura portadores de cargah+: $h_{V B}^{+} \rightarrow h_{T R}^{+}$

Recombinacion electrón - hueco : $e_{T R}^{-}+h_{V B}^{+}\left(h_{T R}^{+}\right) \rightarrow e_{C B}^{-}+$calor

Barrido de e fotoexcitado: $\left(\mathrm{O}_{2}\right)_{\text {ads }}+e^{-} \rightarrow \mathrm{O}_{2}^{\bullet-}$

Oxidación de hidroxilos : $\mathrm{OH}^{-}+h^{+} \rightarrow \mathrm{OH}^{\bullet}$

Fotodegradación por $\mathrm{OH}^{\bullet}: \mathrm{R}-\mathrm{H}+\mathrm{OH}^{\bullet} \rightarrow \mathrm{R}^{\bullet}+\mathrm{H}_{2} \mathrm{O}$

Fotohuecos directos : $R+h^{+} \rightarrow R^{+\bullet} \rightarrow$ Itermediarios/Productos

Protonación de superóxidos : $\mathrm{O}_{2}^{\bullet-}+\mathrm{OH}^{\bullet} \rightarrow \mathrm{HOO}^{\bullet}$

Co-barrido de $e^{-}: \mathrm{HOO}^{\bullet}+e^{-} \rightarrow \mathrm{HO}_{2}^{-}$

Formación de $\mathrm{H}_{2} \mathrm{O}_{2}: \mathrm{HOO}^{-}+\mathrm{H}^{+} \rightarrow \mathrm{H}_{2} \mathrm{O}_{2}$

El electrón de transferencia y hueco de transferencia, representan la banda de valencia y de conducción respectivamente. Esto reporta que los transportadores están sobre la superficie del $\mathrm{TiO}_{2}$ y no se recombinan inmediatamente después de la excitación del fotón [36]. En ausencia de secuestrantes de electrones, el electrón fotoexcitado se recombina con el hueco de la banda de valencia en nanosegundos con la simultánea disipación de calor.

$$
\text { Recombinacion electrón - hueco : } e_{T R}^{-}+h_{V B}^{+}\left(h_{T R}^{+}\right)
$$

Aunque la presencia de secuestrantes de electrones es vital para prolongar la recombinación y exitoso funcionamiento de la fotocatálisis. La presencia de oxígeno puede prevenir la recombinación del par electrón-hueco, permitiendo la formación del radical superóxido $\left(\mathrm{O}_{2}{ }^{-}\right)$.

$$
\text { Barrido de } e^{-} \text {fotoexcitado: }\left(\mathrm{O}_{2}\right)_{a d s}+e^{-} \rightarrow \mathrm{O}_{2}^{\bullet-}
$$

Estos radicales superóxido $\left(\mathrm{O}_{2}{ }^{-}{ }^{-}\right)$pueden ser completamente protonados para formar el radical hidroperoxil $\left(\mathrm{HO}_{2} \cdot\right)$ y subsecuentemente $\mathrm{H}_{2} \mathrm{O}_{2}$.

Protonación de superóxidos : $\mathrm{O}_{2}^{\bullet-}+\mathrm{OH}^{\bullet} \rightarrow \mathrm{HOO}^{\bullet}$ 
Co-barrido de $e^{-}: \mathrm{HOO}^{\bullet}+e^{-} \rightarrow \mathrm{HO}_{2}^{-}$

Formación de $\mathrm{H}_{2} \mathrm{O}_{2}: \mathrm{HOO}^{-}+\mathrm{H}^{+} \rightarrow \mathrm{H}_{2} \mathrm{O}_{2}$

El radical hidroperoxil $\left(\mathrm{HO}_{2} \cdot\right)$ formado también tiene la propiedad de secuestrante, la coexistencia de estas especies radicales puede doblemente prolongar el tiempo de recombinación $\mathrm{de}^{+}{ }^{\mathrm{TR}}$ en la reacción de fotocatálisis. Se puede notar que la fotocatálisis se lleva a cabo gracias a la presencia de oxígeno disuelto (DO) y agua. Sin la presencia de moléculas de agua los altamente reactivos radicales hidroxilo $\left(\mathrm{OH}^{*}\right)$ no pueden ser formados e impide la fotodegradación de compuestos orgánicos presentes en la fase líquida. En la fotocatálisis heterogénea, los compuestos orgánicos que se encuentran en la fase la liquida son degradados a sus correspondientes intermediarios y a una completa mineralización a dióxido de carbono y agua, si el tiempo de irradiación se extiende.

$$
\text { Contaminantes orgánicos } \stackrel{\mathrm{TiO}_{2} / h v}{\longrightarrow} \text { Intermediarios } \rightarrow \mathrm{CO}_{2}+\mathrm{H}_{2} \mathrm{O}
$$

2.5.2. Reacción homogénea de Foto-Fenton La reacción Fenton es un proceso que no involucra radiación comparada con la reacción heterogénea de fotocatálisis donde se emplea $\mathrm{TiO}_{2}$, mientras que el foto-Fenton reacciona a longitudes de onda de $600 \mathrm{~nm}$. Este método es uno de los más aplicados por permitir degradar alta carga de compuestos orgánicos en condiciones de alta salinidad [37]. Existen numerosos estudios de degradación empleando foto-Fenton para clorofenol [32], plaguicidas [38,39], y compuestos orgánicos aromáticos con carga orgánica superior a los $25 \mathrm{~g} / \mathrm{L}$ [40]. En ausencia de una fuente de luz, el peróxido de hidrógeno $\left(\mathrm{H}_{2} \mathrm{O}_{2}\right)$ se puede descomponer por iones de hierro $\left(\mathrm{Fe}^{2+}\right)$ que pueden estar presentes en la fase acuosa (aq), resultando la formación de radicales hidroxilo. La reacción Fenton se puede representar de la siguiente manera:

$$
\mathrm{Fe}^{2+}(a q)+\mathrm{H}_{2} \mathrm{O}_{2} \rightarrow \mathrm{Fe}^{3+}(a q)+\mathrm{OH}^{-}+\mathrm{HO} \bullet
$$

$\mathrm{El} \mathrm{Fe}^{2+}$ se puede revertir a $\mathrm{Fe}^{3+}$ por medio de diferentes mecanismos

$$
\begin{gathered}
\mathrm{Fe}^{3+}(a q)+\mathrm{H}_{2} \mathrm{O}_{2} \rightarrow \mathrm{Fe}^{2+}(a q)+\mathrm{HO}_{2} \bullet+H^{+} \\
\mathrm{Fe}^{3+}(a q)+\mathrm{HO}_{2} \bullet \rightarrow \mathrm{Fe}^{2+}(a q)+\mathrm{O}_{2}+H^{+}
\end{gathered}
$$

Cuando la fuente de luz está presente, el proceso de foto-Fenton se incrementa positivamente comparado con las condiciones de oscuridad. Esto es principalmente debido a la regeneración del $\mathrm{Fe}^{2+}$ (aq) desde el efecto fotoquímico de la luz y la concurrente regeneración del $\mathrm{OH} \cdot$, siempre que la concentración de $\mathrm{H}_{2} \mathrm{O}_{2}$ en el sistema sea considerable. A pesar que el foto-Fenton es altamente fotoactivo en comparación con la catálisis heterogénea, su adecuada operación es ampliamente dependiente de varios parámetros de calidad del agua. Se ha reportado que el pH de 2.8 es el adecuado para llevar a cabo la reacción de foto-Fenton [41].

2.5.3. Avances en inmovilización de fotocatalizadores y soportes Desde que se descubrió el efecto fotocatalítico empleando electrodos de $\mathrm{TiO}_{2}$ [22], numerosos investigadores se han involucrado en la síntesis de catalizadores de $\mathrm{TiO}_{2}$ de diferente escala, realizar la caracterización de sus propiedades físicas y determinar la eficiencia de fotooxidación sobre la superficie, orientada a la naturaleza de la reacción de fotocatálisis $[22,42,43]$. A la fecha, el fotocatalizador más 
ampliamente utilizado ha sido el Degussa P-25. Este catalizador es usado como estándar de referencia para comparar la fotoactividad bajo diferentes condiciones de tratamiento [44]. Las finas partículas de $\mathrm{TiO}_{2}$ de Degussa P-25 siempre han sido aplicadas en la forma de compuesto líquido. Es por esto que el catalizador de $\mathrm{TiO}_{2}$ en forma líquida (slurry) es el catalizador de $\mathrm{TiO}_{2}$ con mayor cantidad de aplicaciones $[45,46]$.

2.5.4. Configuración de un fotoreactor catalítico Los fotoreactores para el tratamiento del agua se clasifican generalmente dependiendo del estado del catalizador: 1) Reactores con partículas fotocatalíticas suspendidas, 2) Reactores con fotocatalizador inmovilizado sobre un soporte inerte continuo. Las primeras planta piloto con reactores para descontaminación solar fueron desarrollados al final de la década de los 80 por los Laboratorios Nacionales Sandía en EE.UU, usando Colectores Cilindro Parabólicos (PTC) para aplicaciones térmicas que fueron modificados simplemente reemplazando el tubo receptor por un tubo pyrex a lo largo del cual fluía el agua contaminada [47]. Los reactores pueden ser diseñados con colectores o sin colectores de radiación. Los sistemas de concentración de radiación tienen la ventaja de tener un área de reactor-tubos menor, lo que puede significar un circuito menor y con mayor facilidad para confinar, controlar y manipular el agua a tratar. Entre las desventajas se encuentran, que no pueden concentrar la radiación difusa, la complejidad, costo y necesidades de mantenimiento y variación de la velocidad de las reacciones fotocatalíticas con respecto a la radiación incidente. Los colectores solares sin concentración son, en principio, más baratos que los PTCs y no tienen partes móviles o mecanismos de seguimiento solar. Los colectores cilindro parabólico compuestos CPCs son una interesante mezcla entre los PTCs y los sistemas sin concentración y suponen una de las mejores opciones para las aplicaciones fotocatalíticas solares [34,48]. Colectores cilindro parabólicos compuestos (CPCs) han permitido obtener porcentajes de decoloración del $100 \%$ para aguas con presencia de colorante azul (RB4) y $82 \%$ de TOC y $23 \%$ de COD fueron alcanzados [49]. Una representación esquemática de un fotorreactor se presenta en la figura 4 .

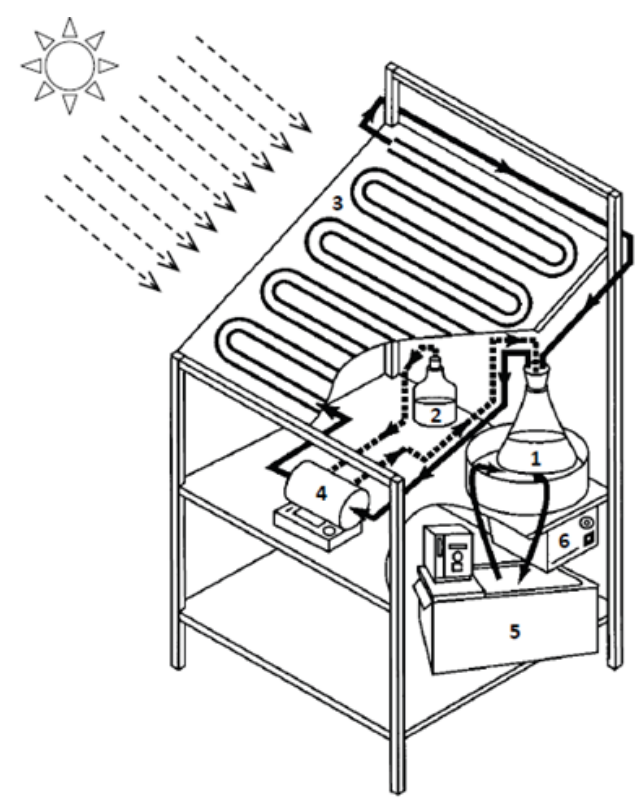

Figura 4: Estación experimental de fotodegradación. (1) Erlenmeyer; Solución de $\mathrm{H}_{2} \mathrm{O}_{2}$; (3) Reactor Solar; (4) Bomba Peristáltica (5) Baño termostático (6) Agitador magnético [50]. 


\subsection{Tratamientos de membrana}

La frecuente detección de muchos plaguicidas en las aguas naturales es de gran preocupación para las autoridades y las poblaciones en general debido a la imperiosa necesidad del agua potable [51]. Donde la presencia de este tipo de sustancias en bajas concentraciones (pg/L a $\mathrm{ng} / \mathrm{L}$ ) en el agua dificulta su tratamiento, más aun si son aguas residuales o de reúso que por lo general tienen asociados efectos adverso sobre la salud humana. Por esto es necesario implementar los controles apropiados de regulación y las mejores prácticas en el uso de plaguicidas, vislumbrándose una necesidad urgente como es la determinación y remoción de plaguicidas presentes en fuentes de agua potable. Esto es una tarea difícil debido a que gran parte de estas sustancias son ampliamente esparcidas en el medio ambiente debido a que son aplicadas para la protección de las cosechas. Los métodos convencionales para el tratamiento de agua potable, que son ampliamente empleados, comprenden coagulación-floculación de partículas, sedimentación y filtración, son poco efectivos para remover residuos de plaguicidas $[52,53]$. La adición de una etapa de tratamiento avanzada (usualmente, involucrando la oxidación con $\mathrm{O}_{3}$, o $\mathrm{H}_{2} \mathrm{O}_{2}$ y filtración con carbón activado granular, son generalmente considerados por ser efectivos, aunque problemas significantes se siguen presentando, principalmente con la saturación del carbón activado y la toxicidad de subproductos formados durante el proceso de filtración [54].

En vista de los problemas que se presentan en los procesos usados actualmente para remover plaguicidas, como también otra gran cantidad de microcontaminantes orgánicos sintéticos frecuentemente encontrados en fuentes de agua potable (Farmacéuticos, productos de cuidado personal, detergentes, subproductos de desinfección, esteroides, disrruptores endocrinos, materiales plastificantes, entre otros), se ha realizado un esfuerzo importante en investigación de métodos de tratamiento efectivos basados en procesos de presión conducidos a través de membrana. Teniendo en cuenta que la mayoría de plaguicidas presentan un peso molecular superior a 200 Da y tamaños en el rango de los iones (cerca de $1 \mathrm{~nm}$ ), normalmente son de gran utilidad el uso de ósmosis reversa (OR) y la nanofiltración (NF) como los más apropiados procesos para remover plaguicidas presentes en fuentes de agua contaminadas.

En los años 60, [55] estudiaron la remoción de unos pocos plaguicidas organoclorados como DDT, TDE, BHC y Lindano por ósmosis reversa (OR) usando una membrana asimétrica de acetato de celulosa. Los resultados iniciales del estudio mostraron que la filtración con OR empleando la membrana de acetato de celulosa, es un prometedor proceso de tratamiento para producir agua con baja concentración de sustancias orgánicas, incluyendo plaguicidas. [56] investigaron la eficiencia de la OR en la remoción de una gran variedad de plaguicidas incluyendo organoclorados, organofosforados y misceláneos, en la cual emplearon membranas aromáticas de poliamida (PA) y membranas de pilietilenimina (con enlazamiento cruzado), presentando mejor eficiencia en la remoción de plaguicidas y mayor resistencia a la acidez que las membranas convencionales de acetato de celulosa (CA). Desde mediados de los años 80, el desarrollo de tecnologías de membrana ha presentado diferentes avances entre los que se encuentran la capacidad del rechazo de sales, estabilidad química y reducción de requerimientos de presión. Entre los avances se encuentran la nanofiltración y el desarrollo de membranas de osmosis reversa de ultra baja presión (ULPRO). Entre las distintas características de las membranas de nanofiltración se encuentran el adecuado ablandamiento del agua (rechazo de iones divalentes y multivalentes) sin cambios significativos en la salinidad del agua, incrementando el rechazo de compuestos orgánicos disueltos no cargados, alcanzando altos flujos de agua a relativas bajas presiones de operación, brindando la mejor opción de tratamiento de aguas para consumo contaminadas con plaguicidas. Un ejemplo para la producción de agua potable eliminando compuestos orgánicos y plaguicidas es la planta Méry-sur-Oise ubicada al norte de Paris, Francia. La planta produce agua de excelente calidad, tomada del rio Oise usando nanotecnología desde el año 1999. Los indicadores de eficiencia son muy satisfactorios principalmente a los relacionados con la eliminación de materia orgánica y plaguicidas, lo cual indica que la nanofiltración puede 
ser exitosamente empleada a gran escala [54].

\subsection{Adsorción}

El proceso de adsorción consiste en la captación de sustancias solubles en la superficie de un sólido. Un parámetro fundamental es este caso será la superficie específica del sólido, dado que el compuesto soluble a eliminar se ha de concentrar en la superficie del mismo. La necesidad de una mayor calidad de las aguas está haciendo que este tratamiento esté en auge. Es considerado como un tratamiento de refino, y por lo tanto usado al final de los sistemas de tratamientos más usuales, especialmente con posterioridad a un tratamiento biológico. El sólido universalmente utilizado en el tratamiento de aguas es el carbón activo, aunque recientemente se están desarrollando diversos materiales sólidos que mejoran, en ciertas aplicaciones, las propiedades del carbón activo. La viabilidad económica de este proceso depende de la existencia de un medio eficaz de regeneración del sólido una vez agotada su capacidad de adsorción. Las propiedades del carbón activo se deterioran, por lo que es necesario reponer parte del mismo por carbón virgen en cada ciclo. El coste es un parámetro importante a la hora de la elección del adsorbente. Alternativas al carbón activo son las zeolitas, arcillas (montmorillonita, sepiolita, bentonita, etc.), los denominados adsorbentes de bajo coste, procedentes en su mayor parte de residuos sólidos orgánicos. Recientemente se están desarrollando derivados de polisacáridos (biopolímeros derivados del almidón) [57]. Las aplicaciones de la operación de adsorción es amplia, desde un amplio abanico de sustancias orgánicas (colorantes, fenol, mercaptanos, etc) hasta metales pesados en todos sus estados de oxidación. El tratamiento de aguas usando tecnologías como la osmosis reversa, intercambio iónico, electrodiálisis, electrólisis puede estar en el rango de 10-450 dólares por metro cubico de agua tratada, mientras que el costo del tratamiento de agua usando la adsorción está en el rango de 5-200 dólares por metro cúbico [58].

La adsorción puede remover contaminantes orgánicos solubles e insolubles. La capacidad de remoción de este método puede estar por encima del $99.9 \%$. Debido a estos altos rendimientos, la adsorción ha sido usada para remover una variedad de contaminantes orgánicos de varias fuentes de agua contaminadas. Básicamente la adsorción es la acumulación de una sustancia sobre una superficie o interface. En el caso de tratamiento de aguas, el proceso ocurre en una interface entre el adsorbente sólido y el agua contaminada. El contaminante que es adsorbido es llamado adsorbato y la fase de adsorción el adsorbente. La adsorción de contaminantes se puede realizar usando materiales orgánicos e inorgánicos. Para el proceso de adsorción es necesario tener en cuenta parámetros como tiempo de equilibrio, $\mathrm{pH}$, efecto del medio iónico, concentración inicial del soluto, entre otros.

\subsection{Nanopartículas}

Las tecnologías de degradación de plaguicidas ahora han avanzado al uso de nanopartículas de varios tipos. Durante las últimas décadas, nanopartículas como el $\mathrm{TiO}_{2}$ y el Fe han demostrado ser excelentes adsorbentes y con alta eficiencia fotocatalítica en la degradación casi completa de plaguicidas organoclorados y sus metabolitos tóxicos, lo cual indica la oportunidad de explorar otro tipo de nanopartículas que pueden ser también empleados en este tipo de procesos. El mal uso y manejo de plaguicidas ha alentado el desarrollo y diseño de métodos eficientes para la remoción de diferentes tipos de plaguicidas, particularmente los organoclorados debido a su carácter recalcitrante. Los nanomateriales han ganado atención en la remoción de diferentes contaminantes presentes en los reservorios de agua, debido a sus propiedades únicas como son: elevada área superficial, baja resistencia a la difusión, alta capacidad de adsorción y alto equilibrio de adsorción [59]. Varios tipos de nanomateriales han sido utilizados para la detección, degradación y remoción de plaguicidas presentes en diferentes matrices. Nanopartículas de Fe, $\mathrm{Ti}, \mathrm{Zn}$ y nanotubos han mostrado una rápida degradación de varios plaguicidas presentes en agua con eficiencias mayores al $90 \%$. Fotocatálisis, decloración y reducción catalítica son los 
mecanismos que explican la degradación por diferentes nanomateriales. Nanopartículas de óxidos de metales y nanotubos han sido utilizados para remover agroquímicos de diferentes matrices vía adsorción o separación magnética. Nanopartículas de óxidos de Zn y nanotubos han mostrado eficiencias de remoción entre el $99 \%$ y el $100 \%$ por su amplia área superficial con química ajustable, tamaño pequeño, alta selectividad y sensibilidad y rápida respuesta son algunas de las ventajas de estos nanomateriales. Sin embargo también presentan algunas desventajas, como el ultra pequeño tamaño de las nanopartículas, que no permite que sean fácilmente recuperadas una vez que quedan dispersas en el agua. Las sustancias químicas empleadas para la modificación de la superficie de diferentes nanomateriales también exhiben efectos tóxicos por exposición al igual que los nanotubos de carbono y de nanopartículas de metales y de óxidos de metales. Biopolímeros como almidón, quitosan y celulosa están siendo empleados como soportes no tóxicos para nanopartículas. La química verde con el empleo de extractos biológicos están reemplazando los químicos tóxicos usados para la funcionalización de la superficie [61]. En la figura 5 se presenta la modificación de nanopartícula de Fe empleando óxido de silicio y su uso para retener plaguicidas presentes en medios acuosos.

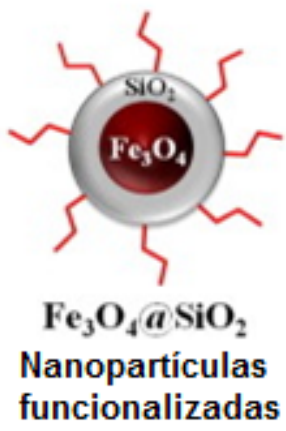

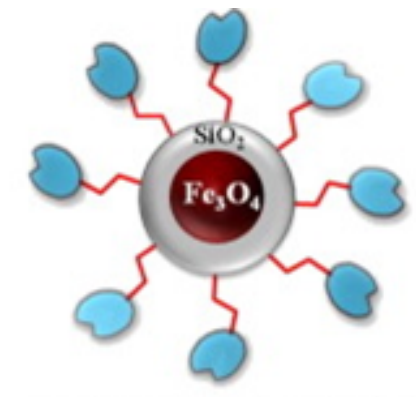

Extracción de plaguicidas

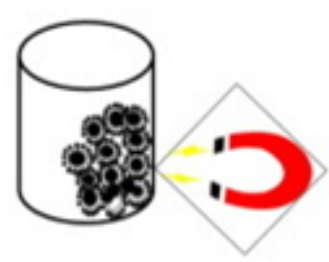

Separación Magnética

Figura 5: Aplicación de nanopartículas de silica magnética para la extracción de plaguicidas [61].

\section{Conclusiones}

El incremento de la conciencia de la sociedad relacionado con el cuidado ambiental, los problemas de salud y los impactos en los ecosistemas de los efluentes industriales y otras corrientes de agua han incentivado el desarrollo de tecnologías de tratamiento del agua. Uno de los cambios que se presentan hoy en día es la eficiencia de remoción de contaminantes orgánicos presentes en cuerpos de agua que pueden ser apenas removidos por métodos convencionales. Las diferentes tecnologías que existen para la remoción de plaguicidas presentes en agua exhiben sus respectivas ventajas y desventajas. Métodos como la precipitación, procesos electroquímicos, intercambio iónico, tratamientos biológicos, procesos avanzados de oxidación, tratamiento de membrana, adsorción sobre diferentes materiales y uso de nanopartículas permiten obtener altos porcentajes de remoción ajustando los parámetros fisicoquímicos necesarios. Los avances científicos y tecnológicos permiten que estas tecnologías continúen mejorando ya sea empleándolas de forma individual o combinando las diferentes tecnologías existentes.

\section{Agradecimientos}

Los autores agradecen a Colciencias y al Departamento de Química de la Universidad del Cauca, Popayán, Colombia, por el apoyo brindado en la realización de este trabajo. 


\section{Referencias}

[1] H. Beitz, H. Schmidt, and F. Herzel, "Occurrence, toxicological and ecotoxicological significance of pesticides in groundwater and surface water". Chem. Plant Prot, vol. 8, pp. 3-53, 1994.

[2] C. Schweizer, T. Turgut and K. H urle. "Untersuchungen zur Verflüchtigung von Pflanzenschutzmitteln von Pflanzenoberflächen in Abhängigkeit von Temperatur und relativer Luftfeuchte" , Zeitschrift für PflKrankh und Pflschutz, vol 17, no.5, pp. 791-798, 2000.

[3] M. Guidottl, R. Giavinazzo, O. Cedrone, M. Vitali, "Determination of organic micropollutants in rain water for laboratory screening of air quality in urban environment" , Environ. Int, vol. 26, pp 23-28, 2000.

[4] T. Nakatani, A. Yamamoto, S. Ogaki, "A survey of dietary intake of polychlorinated dibenzo-p-dioxins, polychlorinated dibenzofurans, and dioxin-like coplanar polychlorinated biphenyls from food during 2000-2002 in Osaka City, Japan" , Archives of Environmental Contamination and Toxicology, 2010. vol 60, pp. 543-555, 2011.

[5] . S.M.Chang, and R.A.Doong, "Concentration and fate of persistent organochlorine pesticides in estuarine sediments using headspace solid-phase microextraction" , Chemosphere, vol. 62, pp.1869-1878, 2006.

[6] .K.L. Willett, E.M. Ulrich, and S.A.Hites, "Differential toxicity and environmental fates of hexachlorocyclohexane isomers", Environ. Sci. Technol, vol 32, pp. 2197, 2207, 1998.

[7] R.A. Doong, and C.Y. Lee, "Determination of organochlorine pesticide residues using solid-phase extraction cleanup cartridges," Analyst, vol. 124, pp. 1287-1289, 1999.

[8] R.A. Doong, C.K. Peng, Y.C. Sun, and P.L. Liao, "Composition and distribution of organochlorine pesticide residues in surface sediments from the Wu-shi River estuary, Taiwan" , Mar. Pollut. Bull, vol. 45, pp. 246-253, 2002.

[9] K. Feng, B.Y. Yu, D.M. Ge, M.H. Wong, X.C. Wang, and Z.H. Cao, "Organochlorine pesticide (DDT and $\mathrm{HCH}$ ) residues in the Taihu Lake Region and its movement in soil-water system I. Field survey of DDT and $\mathrm{HCH}$ residues in ecosystem of the region" , Chemosphere, vol. 50, no. 6, pp. 683-687, 2003.

[10] F. Verweij, K. Booij, K. Satumalay, N. Van Der Molen, R. Van Der Oost, "Assesment of bioavailable PAH, PCB and OCP concentration in wáter, using semipermeable membrane devices (SPMDs), sediments and caged carp" , Chemosphere, vol. 54, pp.1675-1689, 2004.

[11] M.Y. Badii, S. Varela, "Insecticidas organofosforados: Efectos sobre la Salud y el Ambiente" , Culcyt. Toxicología de Insecticidas, vol. 5, no. 28, pp. 5-17, 2008.

[12] J.L. Martinez, M.C. Pablos Espada, A. Garrido Frenich, F.J. Arrebola, "Pesticide trace analysis using solidphase extraction and gas chromatography with electron-capture and tandem mass spectrometric detection in water samples," J. Chromatogr. A, vol. 867, pp.235 -245, 2000.

[13] M.C. Vagi, A.S. Petsas, M.N. Kostopoulou, M.K. Karamanoli, T.D. Lekkas, "Determination of organochlorine pesticides in marine sediments samples using ultrasonic solvent extraction followed by GC-ECD," Desalination, vol. 210, pp. 146-156, 2007.

[14] Y. Lu, Z. Wang, J. Huckins, "Review of the background and application of triolein-containing semipermeable membrane devices in aquatic environmental study" , Aquatic Toxicology, vol. 60, no. 1-2, pp.139-153, 2002.

[15] Y.S. Su, J.F. Jen, "Determination of organophosphorous pesticides in water using in-syringe ultrasoundassisted emulsification and gas chromatography with electron-capture detection" , J. Chromatogr, vol. 1217, no 31, pp.5043-5049, 2010.

[16] G.M.F. Pinto, I.C.S.F. Jardim, "Use of solid-phase extraction and high performance liquid chromatography for the determination of triazine residues in water: validation of the method" , J. Chromatogr. A, vol. 869, pp. $463-469,2000$.

[17] R. Sarria-Villa, W. Ocampo-Duque, M. Paez, M. Schuhmacher, "Presence of PAHs in water and sediments of the Colombian Cauca River during heavy rain episodes, and implications for risk assessment" , Science of the Total Environment, vol 540, pp. 455 - 465, 2016.

[18] M. Rani, U. Shanker, J. Vidhisha, "Recent strategies for removal and degradation of persistent \& toxicorganochlorine pesticides using nanoparticles: A review" , J Environ Manage, vol.190, pp. 208-222, 2017.

[19] D.E. Helbling, "Bioremediation of pesticide-contamined wáter resources: the challenge of low concentrations" , Current Opinion Biotechnology, vol. 33, pp.142-148, 2015.

[20] V.K. Gupta, D. Pathania, S.Agarwal, P. Singh, "Adsorptional photocatalytic degradation of methylene blue onto pectin-CuS nanocomposite under solar light" , Journal of Hazardous Materials, vol.243, pp. 179-186, 2012.

[21] A. Mojiri, J.L. Zhou, B. Robinson, A. Ohashi, N. Ozaki, T. Kindaichi, H. Farraji, M. Vakili, "Pesticides in aquatic environments and their removal by adsorption methods" , Chemosphere, vol. 253, 126646, 2020

[22] A. Fujishima, K. Honda, "Electrochemical photolysis of water at a semiconductor electrode," Nature, vol. 238, pp. 37-38, 1972.

[23] J. Muff, C. Andersen, R. Erichsen, E. Soegaard, "Electrochemical treatment of drainage water from toxic 
dump of pesticides and degradation products" , Electrochimica Acta, vol. 54, pp. 2062-2068, 2009.

[24] E. Brillas, C.A. Martinez-Huitle, "Decontamination of wastewaters containing synthetic organic dyes by electrochemical methods. An updated review" , Appl. Catal. B: Environ, vol. 166-167, pp. 603-643, 2015.

[25] S.G.Segura, J.D. Ocon, M.N. Chong, "Electrochemical oxidation remediation of real wastewater effluents. A review" , Process safety and environmental protection, II vol. 3, pp. 48-67, 2018

[26] H. Humbert, H. Gallard, H. Suty, J.P. Croqué, "Natural organic matter (NOM) and pesticides removal using a combination of ion exchange resin and powdered activated carbon (PAC)", Water Research, vol. 42, pp. $1635-1643,2008$.

[27] L. Zhao, J. Yang, H. YE, F. Zhao AND B. Zeng, "Preparation of hydrophilic surface-imprinted ionic liquid polymer on multi-walled carbon nanotubes for the sensitive electrochemical determination of imidacloprid" , RSC Adv., no. 7, pp. 4704-4709, 2017.

[28] D.E. Helbling, "Bioremedation of pesticide- contaminated wáter resources; the challenge of low concentrations" , Curr Opin Biotechnol, vol. 33. pp. 142-148, 2015.

[29] N. Hamidin, YU. Qiming, D.W. Connell, "Human health risk assessment of chlorinated disinfection byproducts in drinking water using a probabilistic approach," Water Research, vol. 42, no.13, pp. 3264-3274, 2008 .

[30] M. Kerzhentsev, C. Guillard, J.M. Herrmann, P. Pichat, "Photocatalytic pollutant removal in water at room temperature: case study of the total degradation of the insecticide fenitrothion (phosphorothioic acid O,O dimethyl-O-(3-methyl-4-nitro-phenyl) ester)" , Catalysis Today, vol. 27, pp. 215-220, 1996.

[31] S. Esplugas, J. Giménez, S. Conteras, E. Pascual, M. Rodríguez, "Comparison of different advanced oxidation processes for phenol degradation", Water Res, vol. 36, no.4, pp. 1034 -1042, 2002.

[32] M.P. Titus, V. García-Molina, M.A. Baños, J. Giménez, S. Esplugas, "Degradation of chlorophenols by means of advanced oxidation processes: a general review" , Appl. Catal. B: Environ, vol. 47, pp. 219-256, 2004.

[33] A. Vidal, "Developments in Solar Photocatalysis for Water Purification", Chemosphere, vol. 36, no.12, pp. 2593-2966, 1998.

[34] S. Malato, "Optimising Solar Photocatalytic Mineralization of Pesticides by Adding Inorganic Oxidising Species: Application to the Recycling of Pesticides Containers" , Appl. Catal. B: Environ, vol. 28, no. 3-4, pp. 163-174, 2000.

[35] M.N. Chong, B. Jin, C.W.Chow, CL. Saint, "Recent developments in photocatalytic water treatment technology: A review", Water Research, vol. 4, pp. 2997-3027, 2010.

[36] A. Furube, T. Asahi, H. Masuhara, H. Yamashita, M. Anpo, "Direct observation of a picosecond charge separation process in photoexcited platinum-loaded $\mathrm{TiO}_{2}$ particlees by femtosecond diffuse reflectance spectroscopy", Chem. Phys. Lett, vol. 336, pp. 424-430, 2001.

[37] J. Bacardit, J. Stotzner, E. Chamarro, "Effect of salinity on the photo-Fenton process" , Ind. Eng, Chem, Res, vol. 46, pp. 7615-7619, 2007.

[38] H. Fallmann, T. Krutzler, R. Bauer, S. Malato, J. Blanco, "Applicability of the photo-Fenton method for treating wáter containing pesticides" , Catal, Today vol. 54, pp. 309-319, 1999.

[39] P.L. Huston, J.J. Pignatello, "Degradation of selected pesticide active ingredients and commercial formulations in water by the photo-assisted Fenton reaction" , Water Res, vol. 33, no 5, pp. 1238-1246, 1999.

[40] W. Gernjak, M.I. Maldonado, S. Malato, J. Cáceres, T. Krutzler, A. Glaser, R. Bauer, "Pilot-plant treatment of olive mil wastewater (OMW) by solar $\mathrm{TiO}_{2}$ photocatalysis and solar photo-Fenton", Sol. Energy, vol. 77, pp. 567-572, 2004.

[41] J.J. Pignatello, "Dark and photoassisted iron(3)-catalyzed degradation of chlorophenoxy herbicides by hydrogen peroxide", Environ. Sci. Technol, vol. 26, pp. 944-951, 1992.

[42] Y. Kondo, H. Yoshikawa, K. Awaga, M. Murayama, T. Mori, K. Sunada, S. Bandow, S. Lijama, "Preparation,photocatalytic activities, and dye-sensitized solar-cell performance of submicron-scale Ti02 hollow spheres", Langmuir, vol. 24, pp. 547-550, 2008.

[43] E. Hosono, S. Fujihara, K. Kakiuchi, H. IMAI, "Growth of submicrometer-scale reactangular parallelepiped rutile $\mathrm{TiO}_{2}$ films in aqueous $\mathrm{TiCl}_{3}$ solutions under hydrothermal conditions," J. Am. Chem. Soc, vol. 126, pp. 7790-7791, 2004.

[44] N. Serpone, G. Sauvé, R. Koch, H. Tahiri, P. Pichat, P. Piccinini, E. Pelizetti, H. Hidaka, "Standardization protocol of process efficiencies and activation parameters in heterogeneous photocatalysis: relative photonic efficiencies zr" , J. Photochem. Photobiol," A: Chem, vol. 94, pp. 191-203, 1996.

[45] A. Marinas, C. Guillard, J. Marinas, A. Fernández-alba, A. Agüera, J.M. Herrmann, "Photocatalytic degradation of pesticide-acaricide formetanate in aqueous suspension of $\mathrm{TiO}_{2}$ " , Applied Catalysis B: Environmental, vol. 34, pp. 241-252, 2001.

[46] K. H. Wang, Y.H. Hsieh, M.Y Chou, C.Y. Chang, "Photocatalytic degradation of 2-chloro and 2-nitrophenol 
by titanium dioxide suspensions in aqueous solution" , Applied Catalysis B: Environmental, vol. 21, pp.1-8, 1999.

[47] J.V. Anderson, H. Link, M. Bohn, B. Gupta, "Development of U.S. Solar Detoxification Technology: An Introduction", Solar En. Mat, vol. 24, pp. 538-549, 1991.

[48] C. Minero, E. Pelizzetti, S. Malato, J. Blanco, "Large Solar Plant Photocatalytic Water Decontamination: Effect of Operational Parameters" , Solar Energy, vol. 56, no.5, pp. 421-428, 1996.

[49] A. Durán, J.M. Monteagudo, E. Amores, "Solar photo-Fenton degradation of Reactive Blue 4 in a CPC reactor" , Applied Catalysis B: Environmental, vol. 80, no.1-2, pp 42-50, 2008.

[50] Y. Samet, "Fenton and solar photo-Fenton processes for the removal of chlorpyrifos insecticide in wastewater" , Water SA, vol. 38, no. 4, pp. 537-542, 2012.

[51] G.A. Peñuela, D. Barceló, "Application of C18 disks followed by gas chromatography techniques to degradation kinetics, stability and monitoring of endosulfan in wáter" , Journal of Chromatography A, vol. 795 , pp. 93-104, 1998.

[52] H. Jiang, C. Adams, "Treatability of chloro-s-triazines by conventional drinking water treatment technologies" , Water Res, vol. 40, pp.1657-1667, 2006.

[53] M.P. Ormad, N. Miguel, A. Claver, J.M. Matesanz, J.L. Jovelleiro, "Pesticides removal in the process of drinking water production", Chemosphere, vol. 71, pp. 97-106, 2008.

[54] K.V. Plakas, A.J. Karabelas, "Removal of pesticides from wáter by NF and RO membranes - A review," Desalination, vol. 287, pp.255-266, 2012.

[55] H. Hindin, P.J. Bennett, S.S. Narayanan, "Organic compounds removed by reverse osmosis" , Water Sew, works, vol. 116, pp. 466-470, 1969.

[56] E.S.K. Chian, W.N. Bruce, H.H.P. Fang, "Removal of pesticides by reverse osmosis," Environ. Sci. Technol, vol. 9, pp. 52-59, 1975.

[57] G. Crini, "Recent developments in polysaccharide-based materials used as adsorbents in wastewater treatment," Prog polym Sci, vol. 30, pp.38-70, 2005.

[58] I. Ali, M. Asim, T. Khan, "Low cost adsorbents for the removal of organic pollutants from wastewater" , Journal of Environmental Management, vol. 113, pp.170 -183, 2012.

[59] V. Jassal, U. Shanker, S. Gahlot, "Green synthesis of some iron oxide nanoparticles and their interaction with 2-Amino, 3-Amino and 4-Aminopyridines" , Mater. Today Proc, vol. 3, pp.1874-1882, 2016.

[60] D. Rawtani, N. Khatri, S. Tyagi, G. Pandey, "Nanotechnology-based recent approaches for sensing and remediation of pesticides", Journal of Environmental Management, vol. 206, pp. 749-762, 2018.

[61] G. Bapat, C. Labade, A. Chaudhari, S. Zinjarde, "Silica nanoparticle based techniques for extraction, detection, and degradation of pesticides" , Advances in Colloid and Interface Science, vol. 237, pp. 1-14, 2016.
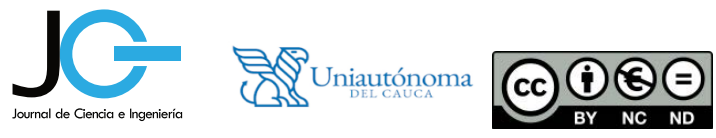